\title{
An Incidental Finding of Heterotopic Pregnancy in a Patient Presented with acute Abdomen post D\&C of Unwanted Pregnancy
}

\author{
Naghem Farouk Abed, ${ }^{1 *}$ Wisam Abdul-Kadder Yassin, ${ }^{1}$ Hayder Assim Mohammed Salih² Aniza Binti Abd Aziz, ${ }^{1}$ Mainul Haque \\ 'Faculty of Medicine, University Sultan Zainal, Jalan Sultan Mahmud, 20400 Kuala Terengganu, Malaysia. \\ 2Senior Specialist in General and Laparoscopic Surgery, Head Manager of the CME dep in Al Karama Teaching Hospital, Baghdad, Iraq.
}

\begin{abstract}
Spontaneous heterotopic pregnancy is a rare condition. There is the simultaneous presence of intrauterine and extrauterine pregnancies. Such condition can be a life-threatening. The diagnosis can also be easily missed especially in early pregnancy. We present this case of a 28-year old patient who was misdiagnosed as normal intrauterine pregnancy. She had illegal termination of unwanted 14-week pregnancy in a private hospital. The patient presented to us with acute abdomen two days after D\&C. Following clinical examination, uterine perforation was suspected. Abdominal ultrasound showed a coexisting abdominal pregnancy in the right hypochondrium. his heterotopic pregnancy was undiagnosed at her initial presentation to the first hospital. Emergency laparotomy was
\end{abstract}

done, uterine perforation was sutured and abdominal pregnancy was excised.

Key words: Heterotopic pregnancy, Intrauterine pregnancy, Abdominal pregnancy, D\&C, Uterine perforation.

Correspondence :

Naghem Farouk Abed

Associate Professor and Obs. \& Gyn. Coordinator, Universiti Sultan Zainal

Abidin, MALAYSIA. Phone no: +601121926620

Email: naghamfa@yahoo.com

DOI: 10.5530/jyp.2016.2.20

\section{INTRODUCTION}

Heterotopic pregnancy is a serious condition that it is difficult to diagnose. ${ }^{1-2}$ It developed very rarely in natural conception, with one of the fertilized egg inside the uterine cavity and another fertilized egg outside the uterus. The location of the extra-uterine pregnancy varies. It can be tubal, abdominal, corneal, cervical or ovarian pregnancy. ${ }^{3-4}$ Fallopian tubes are the most frequent site. Extrauterine pregnancy represents about $1-2 \%$ of all pregnancies with $95 \%$ occurring in the Fallopian tube, ${ }^{5-7}$ while abdominal pregnancy represents just about $1-4 \%$ of all ectopic pregnancies. ${ }^{8-9}$ Most heterotopic pregnancies occur as a result of assisted reproductive technology were it estimated about 1 out of $3900^{10}$ whereas with natural conception the probabilities of heterotopic pregnancy drops to 1 in 30,000 pregnancies. ${ }^{11}$ In the current case, heterotopic pregnancy was misdiagnosed initially as normal intrauterine pregnancy, which was illegally terminated by Dilatation and Curettage (D\&C) at 14 weeks of amenorrhea. This D\&C was complicated by uterine perforation and acute abdomen. In 48 Hospital, Sana'a, Republic of Yemen, an ultrasound revealed a bulky uterus with free fluid in the pouch of Douglas, dated $3^{\text {rd }}$ May 2012. In addition, there was an incidental finding of abdominal pregnancy with an intact gestational sac occupying 13-week dead fetus at the right hypochondrium.

\section{CASE REPORT}

A 28-year-old woman, third gravida brought to the hospital emergency department with abdominal pain associated with vomiting and fever for two days. She had a history of being pregnant of 14 weeks and she went to a private hospital for termination of unwanted pregnancy. D\&C was done and she was discharged home on the same day. The patient felt unwell with abdominal pain which had worsened in the next day. Later on, she developed vomiting three times followed by fever. She did not respond to paracetamol and antibiotic which was prescribed upon discharge.

On examination, she looked severely ill, irritable, toxic and pale. Her pulse rate was $118 / \mathrm{min}$, blood pressure was $100 / 60 \mathrm{~mm} \mathrm{Hg}$, respiratory rate was $25 / \mathrm{min}$ and her temperature was $37.8^{\circ} \mathrm{C}$. The abdomen was mildly distended with diffuse and rebound tenderness. Bowel sounds were reduced. On pelvic examination, the cervix was opened $2 \mathrm{~cm}$ with some blood and clots. The uterus felt bulky of 10 weeks size and was tender. As well, there was fullness in the posterior fornix with non-palpable adnexa.

Her laboratory results showed; WBC $14.00 \times 10^{9} / \mathrm{L}$ with neutrophilia, hemoglobin level was $9.8 \mathrm{gm} / \mathrm{L}$. Her renal function tests, serum electrolytes, liver function tests and coagulation profile were within normal limits. Abdominal ultrasound showed bulky uterus with some retained products of conception. There was a mass of $3 \times 3 \mathrm{~cm}$ at the anterior surface of the uterus with free fluid in pouch of Douglas. Besides, there was an intact gestational sac at the right hypochondrium with a single fetus of 13 weeks. Fetal heart beat was negative (Figure 1). The patient was transferred to the operating theater for emergency laparotomy. Under general anesthesia, the abdomen was opened in layers by midline longitudinal incision. On pelvic cavity exploration, the uterus was about 12 weeks in size with $5 \mathrm{~cm}$ irregular lacerated wounds at the lower part of the anterior border with mild bleeding (Figure 2). Both ovaries and Fallopian tubes were morphologically normal. Pouch of Douglas accumulates about $300 \mathrm{cc}$ blood. Intra-operative diagnosis of perforated uterus was made and uterine cavity was evacuated from the retained product and clots. Then, the edges of the perforation was sutured in two layers by vicryle suture No 0 .

Upon abdominal cavity exploration, a mass was seen in the in the peritoneal cavity adhered to greater omentum below the right hepatic loop. There was an intact gestational sac with $15 \times 8 \mathrm{~cm}$ size, enclosing a dead fetus of 14 weeks (Figure 3 ). Then the gestational sac, placenta with the adhered greater omentum were dissected and excised and the stump was ligated with good homeostasis. Peritoneal wash with normal saline was done and drain was placed in situ at the right iliac fossa. The abdominal closure was done in layers. The patient was given broad spectrum antibiotics; third generation cephalosporine intravenously and metronidazole IV infusion for two days, then proceeded by the oral route for five days. Postoperative follow-up was uneventful apart from one unit of blood was given and the drain removed in the second post-operative day. The patient recovered successfully and was discharged on the seventh postoperative day after stitches removal. 


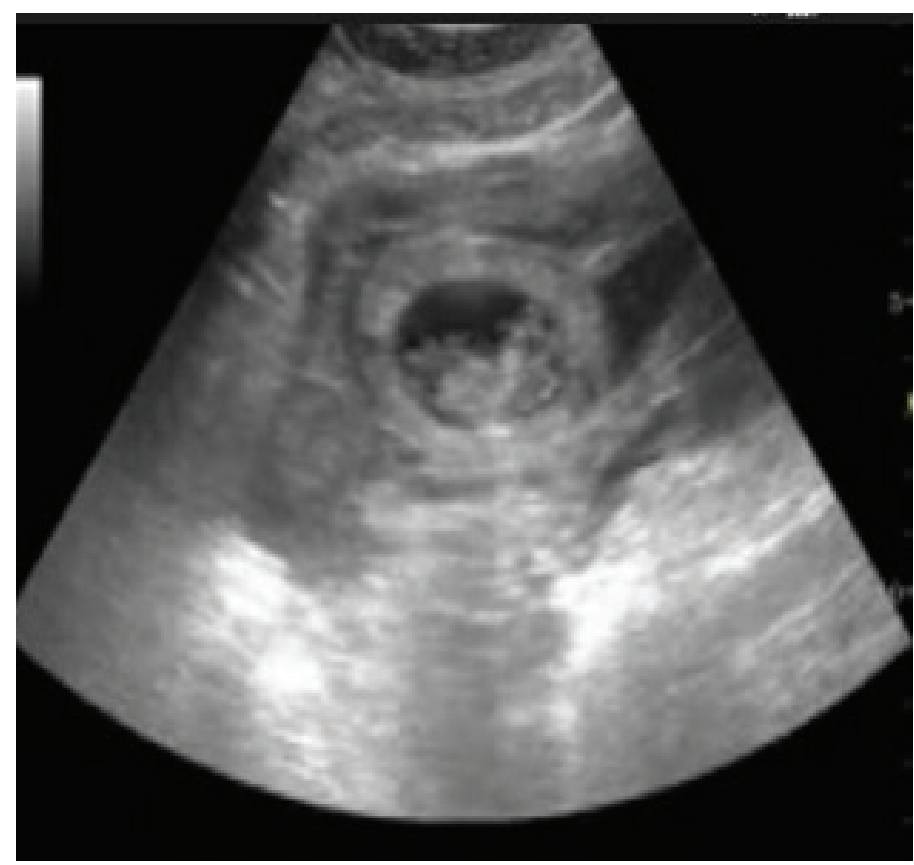

Figure 1: Showing the ultrasound of 13 week abdominal pregnancy

\section{DISCUSSION}

Heterotopic pregnancy is defined as the presence of multiple gestations, with one being intrauterine pregnancy and other extra-uterine pregnancy. The latter is commonly found in the Fallopian tube and uncommonly in the cervix, ovary or abdomen. ${ }^{11-12}$ Heterotopic pregnancy is extremely rare type of pregnancy. The incidence quoted is 1 out of 30,000 pregnancies. ${ }^{11}$ It is also reported to be as high as 1 out of 3900 when the pregnancy is a result of assisted reproductive technology. ${ }^{10,13}$ It's a life-threatening condition and associated with high maternal morbidity and mortality rates..$^{14}$ On the other side, uterine perforation after $\mathrm{D} \& \mathrm{C}$ is also rare $(1.98 \%)^{15}$ and many uterine perforations were unrecognized or asymptomatic ${ }^{16}$ and just treated conservatively. However, serious complications of perforation can happen. A number of studies revealed that small intestine ${ }^{17-19}$, appendix ${ }^{20}$ or omentum ${ }^{21}$ were penetrated the uterine cavity through the uterine perforation and the patient was presented with bleeding, worsening abdominal pain, fever and gastrointestinal symptoms. ${ }^{22}$

In our case, the patient had unwanted 14-week pregnancy and her heterotopic pregnancy was misdiagnosed as normal intrauterine pregnancy in her initial presentation because abdominal pregnancy can pose diagnostic complexity, making a prompt diagnosis difficult and a high index of suspicion is essential especially in low resource centers. ${ }^{6}$ Variable clinical presentation of abdominal pregnancy, such as persistent abdominal pain, painful fetal movements, palpation of an abdominal mass separates from the uterus and vaginal bleeding should raise suspicion. ${ }^{8,23}$ However, our patient did not have any symptoms initially and she underwent $\mathrm{D} \& \mathrm{C}$ in a discrete center. This $\mathrm{D} \& \mathrm{C}$ was complicated by serious perforation and acute abdomen with a concomitant abdominal pregnancy discovered fortunately in our institution. The persistence of the abdominal pregnancy with dead fetus without diagnosis might afterward complicated by peritonitis, sepsis, DIC and pulmonary embolus which ultimately may lead to maternal mortality.

\section{CONCLUSION}

Heterotopic pregnancy is very rare. Diagnosis can be difficult in early pregnancy, especially if intrauterine pregnancy is concomitant with ab-

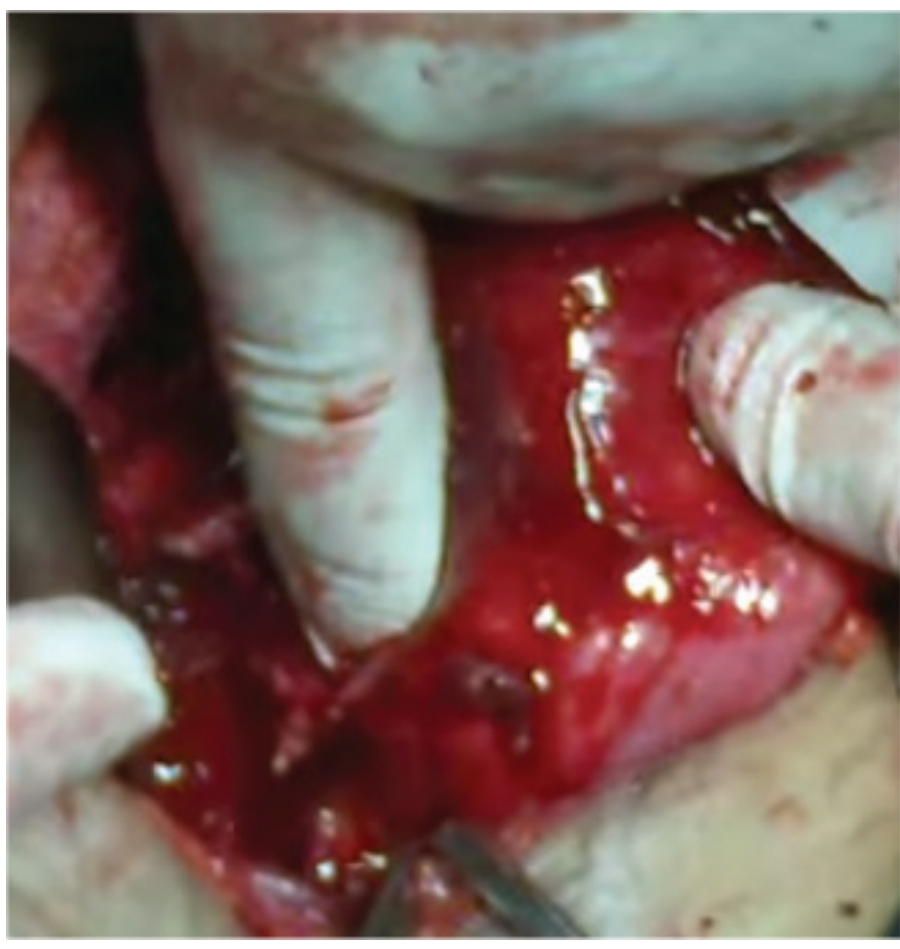

Figure 2: Showing the $5 \mathrm{~cm}$ wound at the lower anterior border of the uterus

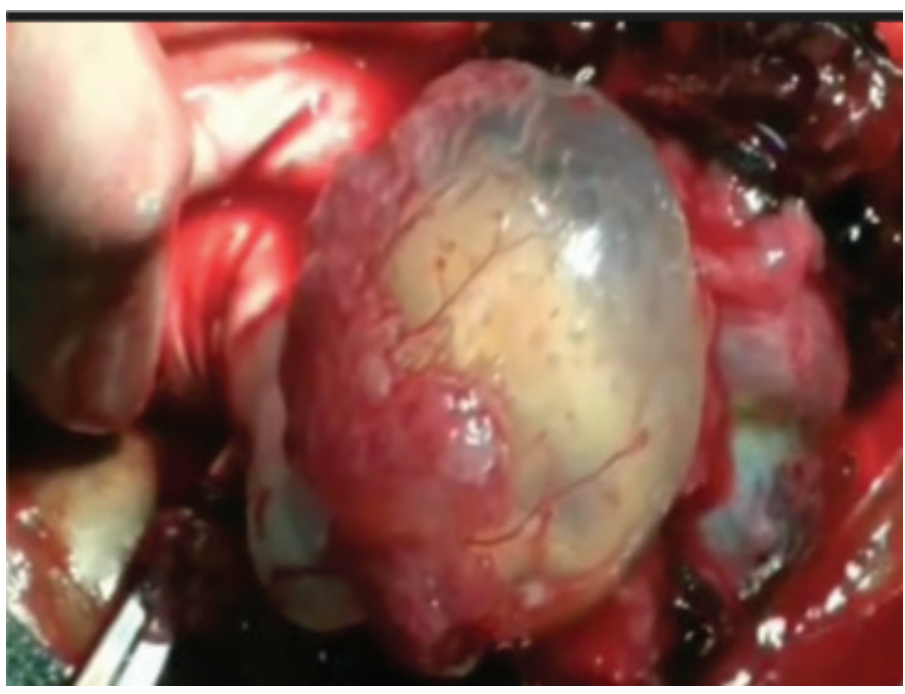

Figure 3: Showing an intact gestational sac with $15 \times 8 \mathrm{~cm}$ size, enclosing a dead fetus of 13 weeks

dominal pregnancy. This needs high suspicion and thorough clinical evaluation with detailed ultrasound examination even though the ultrasound findings depend on the examiner's experience and the quality of the equipment.

A comprehensive abdominal and pelvic ultrasound assessment are recommended for every pregnancy in each trimester even in an asymptomatic pregnant women. Before taking a serious decision of termination of early pregnancy, the physician must carefully evaluate the pregnant woman to determine what risks will be anticipated. Concern should be taken in preparing the patient for $\mathrm{D} \& \mathrm{C}$ so that the risk of uterine perforation can be lessened. D\&C in the second trimester is associated with 
high risk of perforation. Thus, it should be done only if it is crucial, in a well-equipped center and performed by experienced obstetrician. The patient should be kept for at least $24 \mathrm{~h}$ for careful observation before discharge to minimize maternal morbidity.

\section{CONFLICT OF INTEREST}

No financial or other conflict of interest.

\section{ACKNOWLEDGEMENT}

Authors are much greatful to the patient and relatives and Hospital authority.

\section{ABBREVIATIONS USED}

D\&C: Dilatation and Curettage; WBC: White Blood Count; DIC: Disseminated Intravascular Coagulation.

\section{ABOUT AUTHOR}

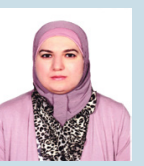

Dr Naghem Farouk Abed Is an Associate Professor and O\&G I Coordinator at University Sultan Zainal Abidin, Faculty of Medicine, 20400 Kuala Terrengganu, Malaysia. She is an O\&G consultant and earned an Arab Board certificate - O\&G (CABOG) - Syria 2008, M.D (O\&G) - Yemen 2009 and FICS (O\&G) - USA 2015. Her area of interest is rare, cases presented in O\&G departments. She has good academic as well as professional experiences since 2008.

\section{REFERENCES}

1. Hassani KIM, Bouazzaoui AEI, Khatouf M, Mazaz K. Heterotopic pregnancy: A diagnosis we should suspect more often. J Emerg Trauma Shock. 2010;3(3):304. doi:10.4103/0974-2700.66563

2. Govindarajan MJ, Rajan R. Heterotopic pregnancy in natural conception. Journa of Human Reproductive Sciences. 2008;1(1):37-8.

3. Basile F, Di Cesare C, Quagliozzi L, Donati L, Bracaglia M, Caruso A, et al. Spontaneous Heterotopic Pregnancy, Simultaneous Ovarian, and Intrauterine: A Case Report," Case Reports in Obstetrics and Gynecology, vol. 2012, Article ID 509694, 4 pages, 2012. doi:10.1155/2012/509694

4. Stucki D, Buss J. The ectopic pregnancy, a diagnostic and therapeutic challenge. Journal of Medicine and Life. 2008;1(1):40-8

5. Hechtman J, Jick B. Heterotopic Pregnancy, Pregnancy corner. 2015. Available from http://www.pregnancycorner.com/loss/heterotopic-pregnancy.html [Accessed on 17-12-2015]

6. Baffoe P, Fofie C, Gandau BN. Term Abdominal Pregnancy with Healthy Newborn: A Case Report. Ghana Med J. 2011;45(2):81-3.

7. Sivalingam VN, Duncan WC, Kirk E, Shephard LA, Horne AW. Diagnosis and management of ectopic pregnancy. J Fam Plann Reprod Health Care. 2011;37(4):231-40

8. Nwobodo El. Abdominal pregnancy: a case report. Ann Afr Med. 2004;3:195-6.

9. Dabiri T, Marroquin GA, Bendek B, Agamasu E, Mikhail M. "Advanced Extrauterine Pregnancy at 33 Weeks with a Healthy Newborn,." Bio Med Research International. 2014;2014: Article ID 102479.

10. Tal J, Haddad S, Gordon N, Timor-Tritsch I. Heterotopic pregnancy after ovulation induction and assisted reproductive technologies: a literature review from 1971 to 1993 . Fertil Steril. 1996;66:1-12.

11. Govindarajan MJ, Rajan R. Heterotopic pregnancy in natural conception. J Hum Reprod Sci. 2008;1(1):37-8.

12. Noor N, Bano I, Parveen S. Heterotopic pregnancy with successful pregnancy outcome. J Hum Reprod Sci. 2012;5(2):213-4.
13. Luo X, Lim CE, Huang C, Wu J, Wong WS, Cheng NC. Heterotopic pregnancy following in vitro fertilization and embryo transfer: 12 cases report. Arch Gyneco Obstet. 2009; 280: 325-9.

14. Mistry BM, Balasubramaniam S, Silverman R, Sakabu SA, Troop BR. Heterotopic pregnancy presenting as an acute abdomen: A diagnostic masquerader. Am Surg. 2000;66(3):307-8.

15. Kaali SG, Szigetvari IA, Bartfai GS. The frequency and management of uterine perforations during first-trimester abortions. Am J Obstet Gynecol. 1989; $161(2): 406-8$.

16. Tristan SB, Gilliam M. First trimester surgical abortion. Clin Obstet Gynecol. 2009;52:151-159.

17. Coughlin LM, Sparks DA, Chase DM, Smith J. Incarcerated small bowel associated with elective abortion uterine perforation. J Emerg Med. 2013;44:e303-6.

18. Augustin G, Majerovic M, Luetic T. Uterine perforation as a complication of surgical abortion-causing small bowel obstruction: a review. Arch Gynecol Obstet. 2013;288(2):311-23

19. Ntia IO, Ekele BA. Bowel prolapse through perforated uterus following induced abortion. West Afr J Med. 2000;19(3):209-11.

20. Dignac A, Novellas S, Fournol M, Caramella T, Bafghi A, Chevallier P. Incarceration of the appendix complicating a uterine perforation following surgical abortion: CT aspects. Emerg Radiol. 2008;15(4):267-9.

21. Ozaki K, Suzuki S. Uterine perforation with omentum incarceration after dilatation and evacuation/curettage. Arch Gynecol Obstet. 2013;287: $607-8$

22. King M, Bewes PC, Cairns J, Thornton J. Primary Surgery, Volume One: Nontrauma. Chapter 8. The surgery of pregnancy. Abdominal pregnancy. Jenna 1999. Available from http://www.meb.uni-bonn.de/dtc/primsurg/docbook/html/ x5173.html [Access date 17-12-2015]

23. Gradzinskas JG. Abdominal pregnancy. In: Edmond OK (ed). Dewhurst's textbook of Obstetrics and Gynaecology for Postgraduates. Blackwell, Edinburgh, 1999. 